\title{
Esophagectomy for Esophageal Cancer in a Patient with Left Pulmonary Artery Sling
}

\author{
Keita Takahashi, MD, Akihiko Okamura, MD (1), En Amada, MD, Reiko Otake, MD, Ryotaro Kozuki, MD, \\ Tasuku Toihata, MD, Yu Imamura, MD, and Masayuki Watanabe, MD
}

Department of Gastroenterological Surgery, The Cancer Institute Hospital of Japanese Foundation for Cancer Research, Tokyo, Japan

\begin{abstract}
Background. In esophageal squamous cell cancer (ESCC) patients, the dissection of the lymph nodes around the recurrent laryngeal nerve (RLN) is essential for curative esophagectomy. ${ }^{1,2}$ Left pulmonary artery sling (LPAS) is a rare congenital anomaly, in which anomalous left pulmonary artery arises from the right pulmonary artery and reach the left pulmonary hilum. ${ }^{3-5}$ Because LPAS crosses between esophagus and trachea and the hemodynamics of LPAS could collapse when retracting the trachea anteriorly for left RLN node dissection, esophagectomy for patients with LPAS is technically challenging. In this video, we applied the cervicothoracoscopic approach in a patient with LPAS, in which we performed bilateral RLN node dissection from cervical operation field before thoracoscopic surgery. ${ }^{6,7}$

Methods. A 44-year-old woman was diagnosed with stage II ESCC. Following neoadjuvant chemotherapy, we planned to perform a three-stage esophagectomy. Preoperativeenhanced computed tomography revealed LPAS. During the cervical procedure, we identified the RLN, dissected the lymph nodes around the nerve, and mobilized the cervical esophagus. After the cervical procedures, we performed thoracoscopic surgery through the right thoracic cavity with the patient in a prone position.
\end{abstract}

Electronic supplementary material The online version of this article (https://doi.org/10.1245/s10434-019-08125-w) contains supplementary material, which is available to authorized users.

(C) Society of Surgical Oncology 2019

First Received: 20 September 2019; Published Online: 12 December 2019

A. Okamura, MD

e-mail: akihiko.okamura@jfcr.or.jp
Results. We achieved curative esophagectomy without any intraoperative adverse events. Total operation time was $419 \mathrm{~min}$, with an estimated blood loss of $40 \mathrm{ml}$. There were no postsurgical complications, including RLN palsy. Conclusions. The presence of LPAS in esophageal cancer surgery makes it difficult to dissect the left RLN nodes. We could safely perform curative esophagectomy for an ESCC patient with LPAS using the cervicothoracoscopic approach.

DISCLOSURES The authors declare no conflicts of interest.

\section{REFERENCES}

1. Matsubara $\mathrm{T}$, et al. Unique distribution patterns of metastatic lymph nodes in patients with superficial carcinoma of the thoracic oesophagus. Br J Surg. 1999;86(5):669-73.

2. Tachimori $Y$, et al. Efficacy of lymph node dissection by node zones according to tumor location for esophageal squamous cell carcinoma. Esophagus. 2016;13:1-7.

3. Wells TR, et al. Reconsideration of the anatomy of sling left pulmonary artery: the association of one form with bridging bronchus and imperforate anus. Anatomic and diagnostic aspects. $J$ Pediatr Surg. 1988;23(10):892-8.

4. Zhong YM, et al. CT assessment of tracheobronchial anomaly in left pulmonary artery sling. Pediatr Radiol. 2010;40(11):1755-62.

5. Yu JM, et al. The prevalence and clinical impact of pulmonary artery sling on school-aged children: a large-scale screening study. Pediatr Pulmonol. 2008;43(7):656-61.

6. Okamura A, et al. Cervicothoracoscopic approach in esophagectomy. Ann Surg Oncol. 2018;25(1):333.

7. Okamura A, et al. Cervicothoracoscopic approach for esophageal cancer in a patient with right-sided aortic arch. Ann Surg Oncol. 2018;25(5):1287.

Publisher's Note Springer Nature remains neutral with regard to jurisdictional claims in published maps and institutional affiliations. 